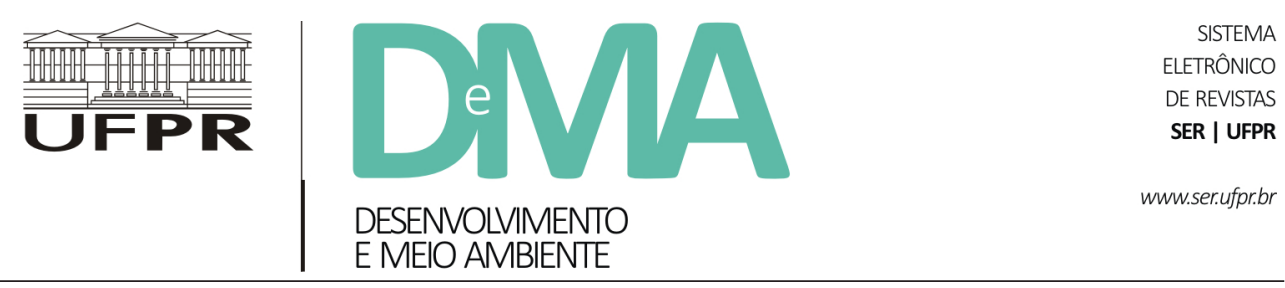

\title{
Simples para ser útil: base ecossistêmica para o gerenciamento costeiro
}

\section{Simple to be Useful: Ecosystem Base for Coastal Management}

\author{
Milton Lafourcade ASMUS ${ }^{1 *}$, João NICOLODI ${ }^{1}$, Marinez Eymael Garcia SCHERER ${ }^{2}$, Kahuam GIANUCA ${ }^{1}$, \\ Julliet Correa COSTA ${ }^{1}$, Lorena GOERSCH${ }^{1}$, Gabriel HALLAL ${ }^{1}$, Kamila Debian VICTOR ${ }^{1}$, Washington L. S. \\ FERREIRA $^{1}$, Júlia N. do A. RIBEIRO ${ }^{1}$, Clara da Rosa PEREIRA ${ }^{1}$, Bruna T. BARRETO ${ }^{1}$, Luciano Figueiredo \\ TORMA $^{1}$, Bruno Bauer G. SOUZA ${ }^{1}$, Marcela MASCARELLO ${ }^{1}$, Allan VILLWOCK ${ }^{1}$
}

\footnotetext{
${ }^{1}$ Universidade Federal do Rio Grande (FURG), Rio Grande, RS, Brasil.

${ }^{2}$ Universidade Federal de Santa Catarina (UFSC), Florianópolis, SC, Brasil.

*E-mail de contato: docamus@furg.br
}

Artigo recebido em 31 de agosto de 2017, versão final aceita em 29 de janeiro de 2018.

RESUMO: Os processos de Gerenciamento Costeiro (GC) nas últimas décadas vêm evoluindo, apresentando diferentes métodos de gestão, sendo que uma nova fronteira se encontra na Gestão com Base Ecossistêmica (GBE). No entanto, para colocar em prática a GBE, a qual leva em consideração as funções, os processos e os serviços ecossistêmicos dos ambientes costeiros e marinhos, entendendo-os como um conjunto de ecossistemas compostos por elementos ecológicos (naturais), econômicos e sociais, se faz necessária a base de informação ecossistêmica. O presente trabalho propõe, apresentando resultados aplicados, um roteiro metodológico de seis etapas: 1. Identificar os ecossistemas como "Unidades de Gestão"; 2. Mapear, modelar e simular os ecossistemas; 3. Identificar e classificar os serviços ecossistêmicos; 4. Definir os valores e a qualidade dos serviços; 5. Identificar os espaços de gestão; e 6. Integrar com políticas e demais instrumentos de gestão e legais. As aplicações práticas apresentadas vão desde trabalhos acadêmicos de identificação e caracterização de ambientes costeiros e marinhos a aplicações nos processos de gestão ambiental portuários, passando também pelo desenvolvimento de Zoneamentos Ecológico-Econômicos (ZEEs) em nível regional, por exemplo. Na apreciação integral do conjunto de exemplos e iniciativas para todas as etapas do modelo, depreende-se que a sua aplicação é ampla, variada e consideravelmente simples. Da mesma forma, a multiplicidade de possíveis aplicações do modelo em ações voltadas ao suporte de uma GBE sugere que seu uso pode crescer e buscar iniciativas inovadoras. A expectativa dos autores é de que tal ferramenta possa, de fato, delinear e estimular pesquisas e aplicações com base ecossistêmica na busca da sustentabilidade da costa e do bem-estar de seus atores sociais. 
Palavras-chave: Gestão com Base Ecossistêmica (GBE); serviços ecossistêmicos; matriz de serviços ecossistêmicos.

ABSTRACT: In the last decades Coastal Management $(\mathrm{CM})$ processes have been evolving, presenting different management methods, and the new frontier is at the so-called Ecosystem Based Management (EBM). However, to put into practice EBM, which takes into account ecosystem functions, processes and services of coastal and marine environments, understanding them as a set of ecosystems composed of ecological (natural), economic and social elements, it is necessary an ecosystem-based information. The present work proposes, presenting practical results, a methodological path of six stages: 1. Identification of ecosystems as "Management Units"; 2. Mapping, modeling and simulating ecosystems and their connections; 3. Identification and classification of ecosystem services; 4. Definition of values and quality of services; 5 . Identification of related management procedures; and 6. Integration with policies and other management and legal tools. The concrete applications range from academic studies of identification and characterization of coastal and marine environments, to port's environmental management processes, and the development of ecological-economic zoning at regional level, for example. In the full appreciation of the set of examples and initiatives for all stages of the model, it can be concluded that its application is wide, varied and considerably simple. Likewise, the multiplicity of possible applications of the model in practical actions aimed to support of an EBM, suggests that its use can grow and pursue innovative initiatives. The authors' expectation is that such a tool may, in fact, delineate and stimulate research and applications based on ecosystems in the quest for the sustainability of the coast and the well-being of its social actors.

Keywords: ecosystem-based management; ecosystem services; ecosystem service matrix.

\section{Introdução}

O Gerenciamento Costeiro (GC) tem apresentado um processo evolutivo nas últimas décadas. Inicialmente, a gestão ambiental de sistemas costeiros esteve predominantemente atrelada às ações de controle de atividades estabelecidas em locais específicos (Burroughs, 2011). São atividades relacionadas à ocupação do espaço costeiro e ao uso de seus recursos com finalidades econômica e social, como a questão do tratamento de efluentes, as atividades de dragagem e as obras de engenharia costeira. É algo que pode ser considerado com Gestão Setorial - focada em um determinado setor ou atividade econômica. Nesse período, o foco para a gestão costeira pode ser traduzido como "licenciamento ambiental", no sentido do controle e da regulação de uma ação local e de seus efeitos na costa. Num segundo momento, a gestão costeira, ao considerar os aspectos espaciais de sua atuação, avança para uma Gestão Espacial. Aqui pode-se tomar como exemplo a definição de unidades de conservação, os estudos sobre ambientes específicos, como manguezais, ou os processos de gestão de uma zona costeira com limites políticos, como a zona costeira de um estado. Nessa considerada gestão espacial, um aspecto-chave é, sem dúvida, o Zoneamento Territorial (ZT). Finalmente e mais recentemente, a crescente necessidade de percebermos, entendermos e, eventualmente, interferirmos em sistemas ambientais complexos, tendo em conta suas características físicas e sociais, tem levado o gerenciamento costeiro a um contexto de Gestão Ecossistêmica (ou Gestão com Base Ecossistêmica - GBE) (McLeod \& Leslie, 2009). É o âmbito em que podem ser geridos sistemas complexos, 
como baías costeiras, estuários, lagoas costeiras. O próprio Planejamento Espacial Marinho (PEM), um instrumento de crescente aplicação em Zonas Econômicas Exclusivas (ZEEs) (Ehler \& Douvere, 2009), tem sua concepção historicamente embasada num enfoque de base ecossistêmica (Blæsbjerg et al., 2009). Talvez o conceito-chave nesse âmbito da gestão costeira seja "serviços ecossistêmicos", uma vez que a GBE trata, de alguma forma, da gestão das atividades humanas, no sentido da manutenção dos serviços ecossistêmicos gerados pelos ecossistemas costeiros (McLeod \& Leslie, 2009) e em seu uso sustentável pela socioeconomia da costa (Dasí, 2011). Neste sentido, a GBE se assemelha e traduz a concepção da Gestão Costeira Integrada, a qual tem diretrizes, metas e objetivos similares à GBE (Barragán, 2014).

A base ecossistêmica para a gestão tem, como concepção fundamental, a consideração de que os sistemas marinhos e costeiros são entendidos como um conjunto de ecossistemas compostos por elementos ecológicos (naturais), econômicos e sociais. Além disso, considera e destaca o caráter funcional dos ecossistemas, capaz de gerar produtos que beneficiam a eles mesmos ou à dinâmica socioeconômica que deles depende (Odum \& Odum, 2001). Os resultados ou produtos dessa funcionalidade de base ecossistêmica, quando geram benefícios aos setores econômicos ou sociais, são considerados Serviços Ecossistêmicos (SE) (MEA, 2003). Os SE têm sido didaticamente agrupados e classificados como (1) de suporte (p. ex., oferecimento de espaço para atividades econômicas), (2) de provisão (p. ex., produção de alimentos), (3) de regulação (p.e. regulação microclimática) ou (4) culturais (p. ex., geração de cenários para contemplação) (de Groot et al., 2002).
Embora sem descartar as ações que envolvem as gestões setoriais e espaciais, a GBE tem se configurado como uma tendência geral no GC no âmbito global (Yáñez-Arancibia et al., 2013; Barragán, 2014). A GBE tem sido proposta, em anos recentes, como uma alternativa aos já tradicionais modelos de gerenciamento costeiro, na busca de uma estrutura de gestão que facilite os processos de integração da informação sobre a zona costeira e possibilite sua efetiva implementação (Agardy et al., 2011). No entanto, embora acatada por âmbitos científicos e administrativos relacionados ao gerenciamento costeiro, os países que tentam adotá-la carecem, em geral, da necessária "base ecossistêmica" que daria suporte a esse modo de gestão (Carollo et al., 2009). No que se refere ao Brasil, há, em geral, um considerável, mas incompleto conhecimento de vários componentes e processos dos ambientes marinhos e costeiros de forma não integrada ou ecossistêmica (Asmus, 2016). Esse conjunto de informações setorizadas, de certa forma, reflete uma característica básica da política ambiental brasileira, na qual importantes instrumentos de planejamento e gestão ambiental - Estudos de Impacto Ambiental (EIA), Planos de Gestão Ambiental (PGA), Zoneamentos Ecológico-Econômicos (ZEE), etc. - são orientados a serem gerados com base em aspectos físicos, biológicos e socioeconômicos, não necessariamente integrados (Asmus, 2016).

Com base nas considerações supramencionadas, o presente trabalho tem como objetivo apresentar uma rota metodológica simples para o estabelecimento de uma base ecossistêmica de ambientes costeiros capaz de suportar ações de gestão com base ecossistêmica e subsidiar avanços nas ações de GC sob diferentes condições ambientais. 


\section{2. $O$ modelo proposto}

O modelo para o roteiro metodológico proposto está concebido como um conjunto de seis etapas, com uma lógica eventualmente conceitual, dependendo da finalidade e da circunstância de sua aplicação. As etapas são as que seguem.

\subsection{Identificação dos ecossistemas como "Unidades de Gestão"}

Tendo em conta a base de informação ecossistêmica para eventuais planejamento e gestão costeira, é importante a identificação dos ecossistemas envolvidos como unidades de gestão. A gestão é realizada tendo em conta o conjunto de ecossistemas, sua condição e seus serviços. Numa versão tradicional, o sistema costeiro seria descrito como o empilhamento de informações físicas, biológicas e socioeconômicas, na expectativa de serem integradas e gerarem possíveis unidades de análise e de gestão. Essa integração tem se demonstrado muito difícil, quando não impossível, levando cientistas ou gestores a optarem por definir um critério de classificação da costa que não necessariamente reflete uma integração da informação considerada. Em ações de Zoneamento Ecológico-Econômico costeiro, por exemplo, os critérios de identificação de unidades de planejamento podem envolver aspectos relacionados com necessidade de preservação ou com níveis de risco para a população (Nicolodi et al., 2017), desconsiderando, pelo menos em parte, os diagnósticos elaborados como informação de base à classificação das zonas (unidades de gestão).

Propõe-se que a identificação dos principais ecossistemas, serviços e benefícios ocorra por meio de uma revisão bibliográfica e com base na opinião especializada de cientistas envolvidos com ecossistemas na área de estudo. Para esse fim, realiza-se uma série de workshops, aplicando princípios de "Opinião Especialista", definidos como procedimentos capazes de capturar o conhecimento de estudiosos em um determinado campo, representando esse conhecimento em uma base de dados, transmitindo-os ao usuário ou pesquisador (Waterman, 1986; Vasconcelos \& Martins-Júnior, 2004). Isso permite obter respostas para perguntas relacionadas à base de conhecimento do sistema em questão. Possíveis inseguranças em torno dos dados produzidos de tal elicitação especializada podem estar relacionadas com incertezas por razões epistemológicas ou por elementos aleatórios, conforme proposto por Regan et al. (2002). Seguindo a sugestão de Martin et al. (2011), a metodologia aqui apresentada trata de reduzir a incerteza, aumentando, dentro do possível, o "tamanho da amostragem" ou o número de participantes no processo de elicitação por especialistas.

\subsection{Mapear, modelar, simular os ecossistemas}

Uma vez identificados, os ecossistemas são mapeados. A Figura 1 é um exemplo de um mapeamento de ecossistemas (Asmus et al., in press) realizado na região conhecida como Baixo Estuário da Lagoa dos Patos (BELP) (Tagliani \& Asmus, 2011), localizado no litoral sul do Rio Grande do Sul.

Eventualmente, para melhor conhecer ou explicitar a sua organização e a sua funcionalidade, os ecossistemas podem ser modelados ou ter seu 
comportamento simulado, conceitual ou matematicamente.

A modelagem conceitual segue a linguagem de modelos ecológicos proposta por Odum (1983), que expressa os ecossistemas como unidades integradas e explicita, de forma clara e simplificada, seus principais elementos, funções, fontes externas e internas, controles de processos, entradas (eg., aportes de energia) e saídas (produtos, por exemplo). Os modelos possuem a vantagem de tornar claros os processos que, eventualmente, geram os serviços ecossistêmicos e as relações de causa e efeitos em relação a sua origem e a sua manutenção, sendo estas últimas fundamentais para identificar possíveis processos ou "espaços" de interferência ou gestão dos serviços de interesse.

\subsection{Identificar e classificar os serviços ecossistêmicos}

A etapa três do roteiro metodológico pode ser considerada como o "coração" do modelo propos-
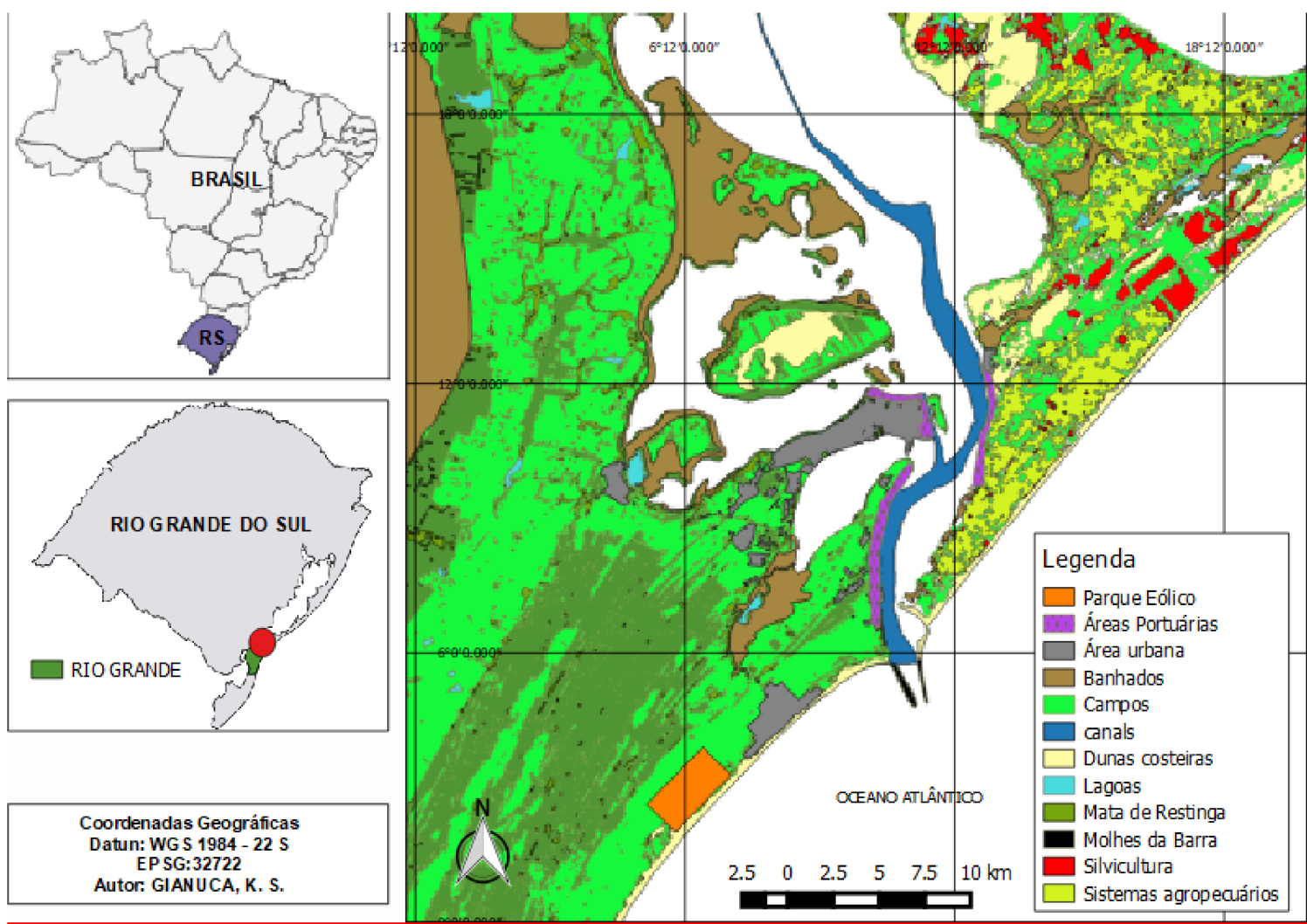

FIGURA 1 - Ecossistemas do Baixo Estuário da Lagoa dos Patos (BELP), RS. FONTE: adaptado de Asmus et al. (in press). 
to. Ela consiste na elaboração de uma Matriz de Ecossistemas e Serviços (MES), com uma estrutura simples e plástica, capaz de adaptar-se a múltiplas análises e aplicações de uso em ecossistemas marinhos e costeiros (Scherer \& Asmus, 2016). Em sua estrutura básica a matriz inclui, para cada ecossistema considerado, os principais serviços ecossistêmicos por eles gerados (classificados como de suporte, de provisão, de regulação ou cultural), os benefícios destacados desses serviços para a dinâmica socioeconômica e quais atores sociais são beneficiados pelos serviços/benefícios. A Tabela 1 apresenta um fragmento da MES elaborada para o Baixo Estuário da Lagoa dos Patos (BELP), focando no ecossistema "marismas". Como o enfoque metodológico é, em última instância, a sua aplicação para o GC, aqueles serviços sem um claro benefício socioeconômico direto não são tratados nas colunas referentes aos "benefícios" e "atores". São serviços considerados como funções ecológicas e que garantem os processos de base e a integridade dos ecossistemas (de Groot et al., 2002). Um exemplo dessas funções na Tabela 1 é a ciclagem de nutrientes nas marismas, classificada como de suporte. Da mesma maneira como desenvolvido na identificação dos ecossistemas, a elaboração da matriz é realizada com base na informação produzida para os ecossistemas considerados no local de análise (revisão bibliográfica) e na aplicação da dinâmica de opinião especialista.

\subsection{Definir valores e qualidade dos serviços}

A definição do valor dos serviços ecossistêmicos não é uma atividade trivial. Embora existam tentativas da definição de um valor monetário (ou de mercado) dos serviços ecossistêmicos (Villasante et al., 2016), o estabelecimento de uma metodologia conspícua para o seu estabelecimento não é entendido como suficiente (TEEB, 2009). Para circundar esse problema, a quarta etapa do modelo trabalha com a percepção de valor por parte dos atores sociais em relação àqueles serviços que lhes beneficiam. Tal percepção procura depreender o

TABELA 1 - Fragmento da Matriz de Ecossistemas e Serviços elaborada para o Baixo Estuário da Lagoa dos Patos (BELP), focando no ecossistema "marismas".

\begin{tabular}{|c|c|c|c|c|}
\hline \multicolumn{5}{|c|}{ Baixo Estuário da Lagoa dos Patos - BELP } \\
\hline \multirow{3}{*}{ Marismas } & Suporte & $\begin{array}{l}\text { Área de refúgio; base para } \\
\text { biodiversidade; berçário na } \\
\text { tural; ciclagem de nutriente }\end{array}$ & & \\
\hline & Provisão & $\begin{array}{l}\text { Produção de biomassa; } \\
\text { fibras vegetais }\end{array}$ & $\begin{array}{c}\text { Pesca artesanal; usos } \\
\text { na agricultura }\end{array}$ & $\begin{array}{l}\text { Pescador artesanal; pequeno } \\
\text { agricultor }\end{array}$ \\
\hline & Cultural & Cenário & $\begin{array}{l}\text { Valor contemplativo; } \\
\text { educação ambiental; } \\
\text { lazer }\end{array}$ & $\begin{array}{l}\text { Comunidade local; turistas } \\
\text { e veranistas; instituições de } \\
\text { ensino; ONGs }\end{array}$ \\
\hline
\end{tabular}


valor relativo dos serviços ecossistêmicos em relação, predominantemente, à atividade econômica (profissional) e ao local de moradia de grupos de atores sociais ou "usuários da costa" (Guermandi et. al., 2009). O valor relativo e percebido é, desta forma, considerado como Valor Social do Serviço (VSS) (Simões et al., 2015). Cabe ressaltar que, ao perceber valores, os atores sociais tendem a incluir o caráter econômico no valor percebido, além de valores menos tangíveis, como o valor afetivo ou o relacionado com aspectos de seu bem-estar social. Adicionalmente, ecossistemas e serviços podem ser valorados por meio da importância relativa que apresentam para as principais atividades econômicas na região considerada. Isso normalmente é feito, nessa etapa, pelo cruzamento das consideradas "macroatividades" com o conjunto de serviços classificados na matriz correspondente. Há, certamente, um valor diferenciado de cada serviço considerado na perspectiva de cada atividade econômica que dele depende e por parte dos responsáveis ou dependentes dessas atividades.

\subsection{Identificar os espaços de gestão}

Essa etapa da rota metodológica parte da premissa de que, em última instância, a GBE em uma zona costeira pode ser expressada com o objetivo de manter os serviços de ecossistemas costeiros, visando ao bem-estar social presente e futuro das comunidades que deles dependem (Yáñez-Arancibia, 2013). Portanto, a partir da identificação, do mapeamento, da valoração social e, destacadamente, da modelagem dos ecossistemas e serviços, é possível identificar os eventuais "espaços de gestão" ou de interferência em fontes, controles ou processos, capazes de influenciar a permanência ou a qualidade dos serviços. Via de regra, a ação para a manutenção dos serviços não ocorre diretamente em um elemento ou em uma função ecológica, mas sim em processos relacionados com o comportamento humano capazes de afetar a funcionalidade dos ecossistemas (controles) (Barragán, 2014). Evidentemente que essa base de informação não completa as necessidades estruturais e processuais da gestão costeira, embora possa ter uma importância fundamental em um contexto que se completa com os múltiplos aspectos da necessária governança para a gestão (Cicin-Sain \& Knecht, 1998).

Quando focada nos espaços para a gestão, a MES pode auxiliar na aplicação de modelos DPSIR (Drivers-Pressure-State-Impact-Response) com base ecossistêmica. O modelo apresenta seu arranjo lógico como Força Motriz-Pressão-Estado-Impacto-Resposta (traduzido do inglês) e pode organizar ações de gestão voltadas à manutenção de ecossistemas costeiros. A MES pode servir de base para o modelo DPSIR e, neste sentido, as Forças Motrizes podem ser interpretadas como macroatividades capazes de gerar efeitos ambientais substanciais nas zonas costeiras, as Pressões como os aspectos ambientais gerados pela ação das forças motrizes, com, por exemplo, uma contaminação da água, o Estado pode ser interpretado como a condição dos ecossistemas frente às pressões ambientais (integridade ou saúde ambiental), os Impactos como impactos nos ecossistemas, capazes de prejudicar ou suprimir serviços ecossistêmicos e, finalmente, as Respostas como ações de gestão para a manutenção de serviços e benefícios. 


\subsection{Integrar com políticas e instrumentos}

A última etapa do modelo envolve a integração da base ecossistêmica de informação com políticas e instrumentos de gestão costeira. Certamente que a desejada integração de uma base de informação ambiental na política de GC envolve uma ação de aproximação e articulação entre ciência e setor governamental (Mee, 2012). No entanto, independentemente desta necessidade, entende-se que a informação com base ecossistêmica, organizada na forma aqui proposta, pode dar apoio a instrumentos de suporte às políticas como Planos de Ordenamento Territorial (POT), Zoneamentos Ecológico-Econômicos Costeiros (ZEEC), Planos de Gestão Ambiental (PGA), Planos Diretores (PD) e Estudos de Impacto Ambiental (EIA). Nesse sentido, um ZEEC poderia ser interpretado, a partir de uma base ecossistêmica, como o ordenamento de ocupação e de uso de ecossistemas costeiros, considerando a potencialidade de seus serviços para as possíveis atividades econômicas e as restrições ou adequações dessas atividades para a manutenção ou o uso sustentável dos serviços considerados. Em uma eventual aplicação a estudos de impactos ambientais (EIAs), ela pode ser interpretada e aqui proposta como "uma avaliação do risco de perder serviços ecossistêmicos (impacto) e seus benefícios para atores sociais identificados, motivado pelo efeito de empreendimentos propostos sobre os mecanismos de manutenção dos serviços". Finalmente, em um plano de gestão ambiental, as ações coordenadas de preservação, de mitigação ou de compensação ambientais podem ser tomadas como de preservação, de mitigação e de compensação de serviços ecossistêmicos. Nesse sentido, ações de compen- sação ambiental devem ser concebidas como ações de compensação de serviços ecossistêmicos, tendo em conta seus benefícios econômicos e sociais e os grupos de atores beneficiados (Asmus, 2016).

As etapas de 1 a 4 no modelo podem ser agrupadas e consideradas como elementos da base de informação ecossistêmica para a gestão. Já as etapas 5 e 6 , de forma agrupada, caracterizam-se como elementos de gestão e governança (Figura 2).

\section{Aplicações e desdobramentos da Matriz de Ecossistemas e Serviços}

Considera-se que o modelo do roteiro metodológico proposto, centrado na Matriz de Ecossistemas e Serviços, possui uma elevada capacidade de aplicações e desdobramentos reais e potenciais. Numa primeira perspectiva, algumas das aplicações consideradas envolvem: caracterização ambiental sistêmica de zonas costeiras, definição da base ecossistêmica de economias costeiras, identificação de usos conflitivos (como conflito por serviços ecossistêmicos), modelagem da base ecossistêmica de macroeconomias, estudos de avaliação de riscos ambientais (como riscos de se perder serviços ecossistêmicos), base sistêmica para o desenvolvimento de modelos DPSIR, estudos de impactos ambientais, base ecossistêmica para gestão ambiental portuária, Zoneamento Ecológico-Econômico Costeiro (ZE$\mathrm{EC}$ ), valoração social dos serviços, identificação de atores e processos participativos, gestão de unidades de conservação (UCs) costeiras com base ecossistêmica, entre outros.

Um exemplo de aplicação da Etapa 1 do modelo (identificação dos ecossistemas) foi realizado na Ilha de Santa Catarina e nos sistemas aquáticos 
adjacentes, a qual faz parte do município de Florianópolis, Santa Catarina. Na ilha, foram identificados como sistemas dominantes os seguintes ecossistemas: manguezais, praias, dunas, laguna, lagoa costeira, costão rochoso, floresta ombrófila, restinga, ilhotas, baías, marinho adjacente, sistemas rurais, sistemas de transição rural/urbano, zonas urbanas e aterros tecnogênicos (Asmus \& Scherer, 2015). Note-se que aqui é adotado o conceito de ecossistema para sistemas ambientais com níveis amplamente diferenciados de antropização. São sistemas com típicos componentes ecológicos (ou naturais), econômicos e sociais (Scherer \& Asmus, 2016). Um mapeamento dos ecossistemas dominantes (Etapa 2) considerados para a Ilha de Santa Catarina foi desenvolvido por Lima e colaboradores (2018, in press).

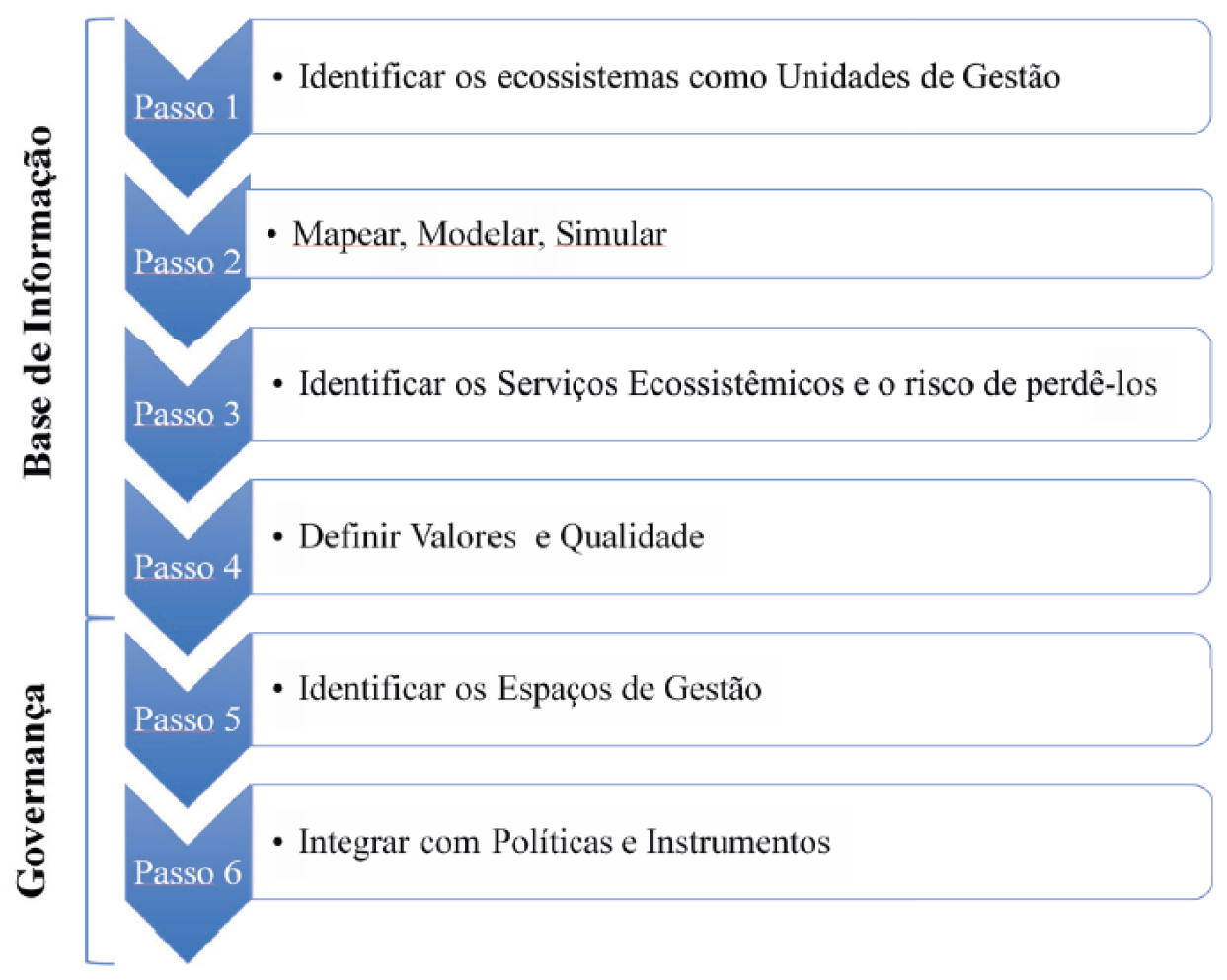

FIGURA 2 - Esquema do roteiro metodológico e agrupamento de etapas. 
Ainda como um exemplo de aplicação da Etapa 2 do modelo, Niehues (2014) modelou a estrutura funcional da base ecossistêmica da Ilha de Santa Catarina, conforme os ecossistemas identificados na Etapa 1 (Figura 3).

A construção da Matriz de Ecossistemas e Serviços (Etapa 3) tem sido implementada em al- guns sistemas costeiros, com destaque para o Baixo Estuário da Lagoa dos Patos (BELP) (Brezolin et al., 2014), Sistema Ilha de Santa Catarina (SISC) (Scherer \& Asmus, 2016) e Laguna de Rocha, Uruguai (Conde et al., 2015), entre outros locais.

Com o uso da matriz para o Baixo Estuário da Lagoa dos Patos, Costa \& Asmus (dados não pu-

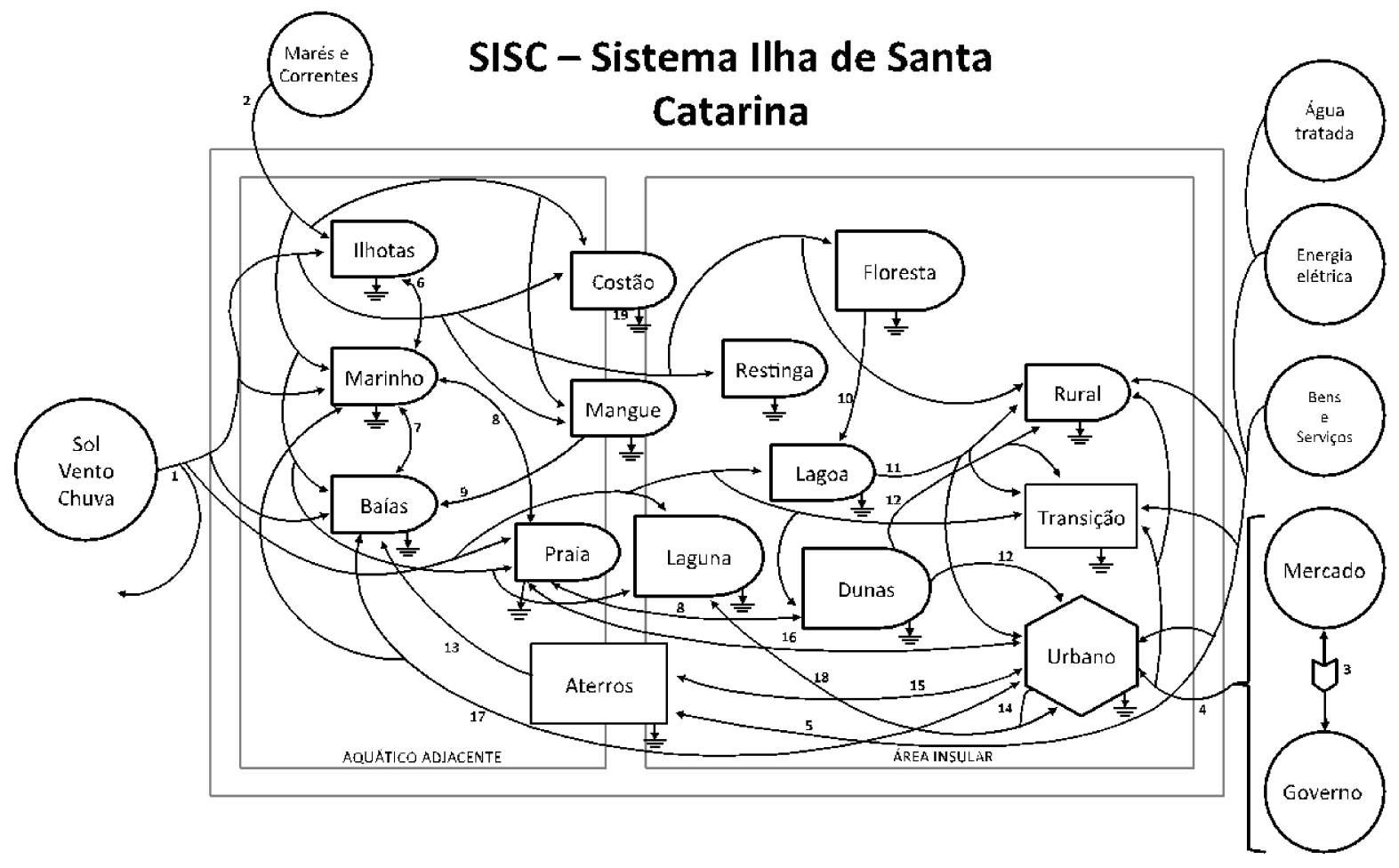

FIGURA 3 - Modelo do Sistema Ilha de Santa Catarina (SISC), com relação funcional entre os ecossistemas dominantes. Numeração dos processos: (1) Energia a partir das fontes renováveis de/para todos os ecossistemas produtores; (2) Transferência de energia das marés e correntes para os ecossistemas que recebem influência do mar; (3) Interação entre controles exercidos pelo Governo e pelo Mercado; (4) Controle do Governo e do Mercado sobre os ecossistemas urbanos, rurais e de transição; (5) Aporte energético vindo de outros produtos externos ao SISC; (6) Fluxo/ Troca de organismos entre as ilhotas e o ecossistema marinho; (7) Pesca (duas vias) e abastecimento marinho; (8) Balanço de sedimentos; (9) Depósito de matéria orgânica; (10) Escoamento/Fornecimento de água; (11)Abastecimento de água; (12) Abastecimento de água; (13) Despejo de resíduos; (14) Diluição; (15) Mobilidade de pessoas; (16) Fluxo de pessoas; (17) Pesca, no sentido Baía/Marinho-Urbano e, no caminho inverso, transferência de resíduos; (18) Pesca, no sentido Laguna-Urbano e, no caminho inverso, transferência de resíduos; (19) Dissipação de energia. FONTE: Niehues (2014). 
blicados) descrevem a base ecossistêmica da pesca artesanal no estuário, utilizando, como exemplo, a pesca da tainha (Mugil liza). Esta análise permitiu a modelagem deste tipo de pescaria e a demonstração dos principais controles e processos da atividade e de sua dependência de típicos ecossistemas estuarinos, além do ecossistema marinho adjacente, a exemplo do modelo utilizado na Ilha de Santa Catarina (Figura 3).

Segundo os estudos no BELP, os serviços ecossistêmicos que proporcionam alguma forma de benefício à atividade pesqueira, identificados de acordo com a sua classificação - suporte, provisão, regulação e cultural (MEA, 2003), foram: (1) Suporte: área de refúgio para o estoque; berçário; base para biodiversidade; ciclagem de nutrientes; espaço para pesca; navegabilidade; corredor ecológico; atracadouro para embarcações; espaço para ocupação (moradia) dos pescadores; (2) Provisão: produção de biomassa; produção de fibras vegetais; (3) Regulação: diluição de poluentes; balanço hidrológico; estabilização para navegação; (4) Culturais: Geração de cenário; relações sociais; reprodução cultural; valor contemplativo.

Martins (2016) também desenvolveu trabalho com a atividade de pesca da tainha para a Ilha de Santa Catarina, identificando todos os ecossistemas envolvidos no fornecimento do serviço ecossistêmico de provisão para essa atividade econômica. Os ecossistemas identificados foram o sistema estuarino da Lagoa da Conceição, como região de berçário da espécie; o ambiente marinho adjacente à Ilha de Santa Catarina; as praias e a zona urbana, que têm importante papel na cadeia produtiva e econômica da atividade.

Em uma análise prospectiva sobre o valor dos serviços ecossistêmicos no Baixo Estuário da Lagoa dos Patos, Simões et al. (2015) utilizaram a MES para não apenas estimar o valor social dos serviços (Etapa 4 do modelo), mas também para avaliar a vulnerabilidade dos diferentes atores sociais em relação às possibilidades de perdê-los em função dos efeitos da mudança climática global em zonas costeiras. Para tal, desenvolveram um sistema de entrevistas sobre a percepção de serviços, benefícios e seus valores relativos em 19 localidades da região. No caso em análise, os efeitos da mudança climática estão predominantemente representados pela frequência crescente de eventos climáticos extremos, como chuvas e ventos intensos, além da ocorrência de granizo (Machado, 2014).

As entrevistas demonstram que, na percepção dos atores sociais, as habitações e os meios de trabalho (principalmente relacionados à pesca artesanal $\mathrm{e}$ à agricultura familiar) seriam mais vulneráveis aos eventos climáticos que afetam diretamente o BELP. No entanto, também demonstram que, na tentativa de manter os serviços de interesse, há várias iniciativas de adaptação por parte das comunidades avaliadas (Simões et al., 2015).

Ainda como um exemplo de aplicação da Etapa 4 do modelo, no que se refere ao valor dos serviços e ao eventual risco em perdê-los, Asmus et al. (2015), após uma simplificação do modelo de Lozoya et al. (2011), analisaram o risco da perda dos serviços ecossistêmicos no BELP, frente às mudanças climáticas, para vários atores sociais. $\mathrm{O}$ modelo utilizado calcula "níveis de risco" como uma função das "ameaças" (intensidade de eventos extremos), "valor dos serviços" (para diferentes atores sociais) e a "vulnerabilidade de cada grupo de atores sociais". Para a elaboração do modelo e a estimativa de risco sob diferentes condições e para diferentes grupos sociais, foram utilizadas as informações a 
partir da MES, além das informações sobre o valor social dos serviços e a vulnerabilidade de cada grupo, com a aplicação da informação gerada por Simões et al. (2015). Uma vez que todos os valores dos fatores do modelo de risco são normatizados (assumindo valores entre 0 e 1), é possível comparar riscos de perda de serviços e benefícios para várias situações. Pode-se trabalhar com cenários de risco para diferentes grupos de serviços, grupos sociais ou mesmo de ecossistemas sob eventuais ameaças, o que permite um possível desenho direcionado a uma gestão com base ecossistêmica, capaz de manter a sustentabilidade dos serviços de interesse no estuário (Asmus et al., 2015).

Outro exemplo de identificação dos serviços ecossistêmicos que dão base aos usos e atividades humanas é o de Figueiredo (2016), que descreveu os Terrenos de Marinha na Ilha de Santa Catarina identificando e caracterizando os sistemas ambientais dominantes, associando os serviços ecossistêmicos destas áreas, determinando os seus usos e os atores envolvidos, bem como a relação dos serviços ecossistêmicos com as atividades e os conflitos dominantes. O estudo analisou os terrenos de marinha como um conjunto de ecossistemas que são responsáveis por gerar serviços ecossistêmicos que dão base às atividades socioeconômicas realizadas, além de demonstrar que a principal causa de conflitos se dá em virtude da disputa de serviços.

Avançando da fase relativa à base de informação (Etapas 1 a 4) para a de gestão e governança (Etapas 5 e 6) do roteiro metodológico, apresentam-se aqui algumas aplicações.

Por exemplo, para estabelecer um subsídio com base ecossistêmica para a estruturação de um sistema de gestão ambiental para o Porto de Imbituba, em Santa Catarina, Scherer et al. (2015) partiram da Matriz de Ecossistemas e Serviços para a região do porto a fim de estabelecer as necessárias diretrizes de gestão. Essa iniciativa, exemplo claro da Fase 5 do modelo (Identificar os Espaços de gestão), culminou com recomendações de uma Gestão Ambiental Portuária Sistêmica (GAPS) por meio da aplicação de um modelo DPSIR vis-à-vis um modelo de melhoria contínua para o porto.

Na metodologia proposta por Scherer, Asmus \& Onetti (2015), também explicada em Onetti et al. (2018), há uma correspondência de base ecossistêmica no modelo DPSIR, no sentido de que as Forças Motrizes correspondem às macroatividades desenvolvidas no sistema portuário (eg., dragagem, transbordo), as Pressões correspondem aos principais aspectos ambientais gerados pelas macroatividades (eg., contaminação da água), o Estado corresponde à condição dos ecossistemas do sistema portuário, os Impactos correspondem às eventuais perdas de serviços ecossistêmicos (como identificados na matriz) e a Resposta pode ser vista como as ações do sistema de gestão ambiental para a manutenção dos serviços ecossistêmicos de interesse para a operação portuária e a comunidade do entorno. Uma aplicação equivalente da MES em sistemas ambientais portuários, com enfoque em um modelo de gestão, foi desenvolvida por Andrade et al. (dados não publicados) para o Porto de São Francisco do Sul, Santa Catarina, com resultados equivalentes.

Finalmente, para exemplificar uma aplicação da Fase 6 do modelo (Integrar com Políticas e Instrumentos), pode-se considerar o fato de que o desenvolvimento metodológico do Zoneamento Ecológico-Econômico (ZEE) do Estado do Rio Grande do Sul (relativo ao ano de 2017) optou por definir como unidade fundamental de planejamento 
territorial os sistemas ambientais (ecossistemas), aplicando os procedimentos estabelecidos na elaboração da MES. Além do uso de técnicas de opinião de especialistas para a identificação e a caracterização dos sistemas (Martin et al., 2011), foram necessárias algumas modificações de nomenclatura, ajustando às classes de uso e de cobertura do solo e a alguns aspectos legais. Os sistemas considerados para o Rio Grande do Sul, na escala do ZEE (1:100.000), foram Afloramentos Rochosos, Sistemas Lóticos, Sistemas Lênticos Interiores, Lagunas e Lagoas Costeiras, Sistema de Praia e Duna Costeira, Sistema de Praia e Duna Lagunar, Areias com Influência Aluvial, Sistema de Áreas Úmidas, Campos Associados à Pecuária, Sistema Agropecuário, Mata Ciliar, Mata de Restinga, Floresta Semidecidual, Floresta Decidual, Sistema de Silvicultura, Sistema Urbano, Sistema Industrial, Sistema Aquaviário e Sistema Viário Terrestre (Silveira et al., 2017).

\section{Considerações finais - Por que simples para ser útil?}

Esse trabalho origina-se da premissa e da percepção, por parte dos autores, de que o modelo amplamente adotado de compilação da informação sobre as zonas costeiras como uma base de informação de suporte ao gerenciamento tem se demonstrado inadequado e insuficiente. Nesse sentido, propõe uma alternativa que envolve não somente uma concepção lógica de como caracterizar e expressar o ambiente costeiro por meio de um enfoque sistêmico, mas, para além disso, demonstra a aplicação desta proposta em uma variedade de situações em ambientes costeiros diferenciados.
A exemplificação das seis etapas do roteiro metodológico proposto, com resultados concretos de sua consecução, serve como um indicador positivo de sua aplicabilidade e seu estímulo para o seu refinamento e aprofundamento. Nesse sentido, parece importante o fato de que o modelo sugerido tem na simplicidade um caráter dominante. Em geral, os procedimentos propostos e desenvolvidos em todos os casos aqui exemplificados são de execução clara, descomplicada, rápida e de baixo custo. Evidencia, portanto, uma interessante utilidade no sentido do estabelecimento de uma plataforma de suporte ao Gerenciamento Costeiro com base ecossistêmica.

$\mathrm{Na}$ visão dos autores, a facilidade utilizada na metodologia traz as vantagens de se utilizar um enfoque sistêmico em contraste com um possível enfoque setorizado e reducionista. Por outro lado, o modelo, sob hipótese alguma, desqualifica a informação especializada e minuciosa sobre os ambientes costeiros. O modelo parte de uma concepção e uma percepção sistêmica dos ambientes costeiros (seus ecossistemas), mas necessita das informações sobre os aspectos físicos, biológicos e socioeconômicos para detalhar seus componentes e processos (funções).

É evidente que o modelo merece e necessita ser mais bem desenvolvido e há claros espaços para tal. A MES irá sempre refletir as melhores informação e concepção possíveis sobre o papel dos ecossistemas em uma região costeira para seus setores econômicos e sociais. As caracterizações dos serviços produzidos, dos benefícios a partir deles e dos atores envolvidos são fatores que podem ser permanentemente melhorados com o avanço da informação obtida. Da mesma forma, a valoração dos serviços considerados que, até o momento, têm sua avaliação predominantemente embasada 
na percepção de atores sociais por eles afetados, pode gradativamente incluir novas metodologias que introduzam valores de outra ordem, como, por exemplo, valores monetários.

Prováveis aplicações da valoração dos serviços classificadas por grupos sociais envolveriam os Estudos de Impacto Ambiental e questões de compensação ambiental. Nos primeiros, a proposta de que um empreendimento é capaz de gerar produtos e serviços de valor a ser considerado e que pode ser comparado com o valor de serviços e benefícios eventualmente depreciados ou perdidos com a sua implantação. Da mesma maneira, possíveis compensações ambientais, motivadas por danos em ecossistemas e seus serviços, deveriam, idealmente, ser realizadas por meio da "compensação de serviços". Note-se que, pela observação da Matriz de Ecossistemas e Serviços, os beneficiários de serviços considerados são atores sociais relacionados a tipologias características de serviços ecossistêmicos. Logo, não bastaria apenas compensar os serviços perdidos, mas, sim, aqueles perdidos pelos grupos sociais afetados (ou prejudicados).

$\mathrm{Na}$ apreciação integral do conjunto de exemplos e iniciativas para todas as etapas do modelo, depreende-se que sua aplicação é ampla, variada e consideravelmente simples. Da mesma forma, a multiplicidade de possíveis aplicações do modelo em ações voltadas ao suporte de uma gestão com base ecossistêmica sugere que seu uso pode crescer e buscar iniciativas inovadoras. A expectativa dos autores é de que tal ferramenta possa, de fato, delinear e estimular pesquisas e aplicações com base ecossistêmica na busca da sustentabilidade da costa e do bem-estar de seus atores sociais.

\section{Referências}

Agardy, T.; Davis, J.; Sherwood, K.; Vestergaard, O. Taking Steps toward Marine and Coastal Ecosystem-Based Management: An Introductory Guide. UNEP Regional Seas Reports and Studies, 189, 2011.

Andrade, L. G.; Asmus, M. L.; Onetti, J. G.; Scherer, M. E. G. Aplicação da base ecossistêmica na gestão ambiental de portos (dados não publicados).

Asmus, M. L. Gestão com Base Ecossistêmica: Novas possibilidades no Brasil. In: Anais do II Congresso Ibero-Americano sobre Gestão Integrada de Áreas Litorais. Florianópolis, Brasil, 2016.

Asmus, M. L.; Nicolodi, J.; Anello, L. Avaliação das Ameaças sobre os Serviços Ecossistêmicos Considerados. In: Conde, D.; Polette, M.; Asmus, M. Risk, perception and vulnerability to Climate Change in wetland dependent coastal communities in the Southern Cone of Latin America. Final Report, IDRC Climate Change and Water Program, Project 6923001, 2015.

Asmus, M. L.; Nicolodi, J.; Anello, L. S.; Gianuca, K. The risk to lose ecosystem services due to climate change: A South American case. Ecological Engineering, in press. doi: 10.1016/j.ecoleng.2017.12.030

Asmus, M. L.; Scherer, M. E. G. Gestão com Base Ecossistêmica como uma nova etapa no caminho do Gerenciamento Marinho e Costeiro Integrado no Brasil. In: Anais do XVI Congreso Latinoamericano de Ciencias del Mar - COLACMAR. Santa Marta, Colômbia, 2015.

Barragán, J. M. Política, gestión y litoral: una nueva visión de la gestión integrada de áreas litorales. Madrid, ES: Editorial Tébar Flores, 2014. 685 p.

Blæsbjerg, M.; Pawlak, J. F.; Sørensen, T. K.; Vestergaard, O. Marine Spatial Planning in the Nordic region: Principles, Perspectives and Opportunities. Nordic Council of Ministers, 2009.

Brezolin, P. T.; Mascarello, M.; Asmus, M. L. Categorização dos ecossistemas e seus serviços presentes no Baixo Estuário da Lagoa dos Patos, RS. In: Anais do Congresso Brasileiro de Oceanografia. Itajaí, 2014. 
Burroughs, R. Coastal Governance. Washington: Island Press, 2011.

Carollo, C.; Reed, D. J.; Ogden, J. C.; Palandro, D. The importance of data discovery and management in advancing ecosystem-based management. Marine Policy, 33, 651-653, 2009.

Cicin-Sain, B.; Knecht, R. W. Integrated coastal management: Concepts and practices. Washington: Island Press, 1998.

Conde, D.; Polette, M.; Asmus, M. L. Risk, perception and vulnerability to Climate Change in wetland dependent coastal communities in the Southern Cone of Latin America, Final Report, IDRC Climate Change and Water Program, Project 6923001, 2015.

Costa, J.; Asmus, M. L. Base ecossistêmica da atividade pesqueira artesanal: estudo de caso no Baixo Estuário da Lagoa dos Patos (BELP), RS, Brasil. Dados não publicados.

Dasí, J. F. (Coord.). La gestión integrada de zonas costeras. Algo más que una ordenación del litoral revisada? Universitat de València, 2011.

De Groot, R.; Wilson, A.; Boumans, R. M. J. A typology for the classification, description and valuation of ecosystem functions, goods and services. Ecological Economics, 41, 393-408, 2002.

Ehler, C.; Douvere, F. Marine Spatial Planning: a step-by-step approach toward ecosystem-based management. Intergovernmental Oceanographic Commission and Man and the Biosphere Programme. IOC Manual and Guides No. 53, ICAM Dossier No. 6. Paris: UNESCO, 2009.

Figueiredo F. S. Análise sistêmica dos Terrenos de Marinha: caracterização da Ilha de Santa Catarina, Florianópolis-SC. Florianópolis, Trabalho (Conclusão de Curso em Oceanografia) - Universidade Federal de Santa Catarina, 2016.

Guermandi, A.; Portela, P. N.; Rao, N.; Teelucksingh, S. S. Recreational, Cultural and Aesthetic Services from Estuarine and Coastal Ecosystems. Fondazione Eni Enrico Mattei, 2009. Disponível em: <www.feem.it>.

Lima, A. S., Figueiroa, A. C., Gandra, T. B. R., Perezi, B. H.M., Santos, B. A. Q., Scherer, M.E.G. Informação de base ecossistêmica como ferramenta de apoio à gestão costeira integrada da Ilha de Santa Catarina, Brasil (dados não publicados).

Lozoya J.; Sardá, R.; Jiménez, J. A. A methodological framework for multi-hazard risk assessment in beaches. Environmental Science \& Policy, 14, 685, 2011.

Machado, A. A. Estudo dos padrões atmosféricos sinópticos geradores de eventos extremos de altura de onda, intensidade de vento, marés meteorológicas e erosão na costa do Rio Grande do Sul. Rio Grande, Tese (Doutorado) - Programa de Pós-Graduação em Oceanografia Física, Química e Geológica. Universidade Federal do Rio Grande, 2014.

Martin, T. G.; Burgman, M. A.; Fidler, F.; Kuhnert, P. M.; Low-Choy, S.; Mcbride, M. Eliciting Expert Knowledge in Conservation Science. Conservation Biology, 26, 2938, 2011.

Martins, C. A pesca da tainha no sistema ambiental da Ilha de Santa Catarina, Florianópolis - SC. Florianópolis, Trabalho (Conclusão de Curso em Oceanografia) - Universidade Federal de Santa Catarina, 2016.

McLeod, K.; Leslie, H. Ecosystem-based management for the oceans. Washington: Island Press, 2009.

MEA - Millennium Ecosystem Assessment. Ecosystems and human well-being: a framework for assessment. Washington, DC: Island Press; 2003.

Mee, L. Between the Devil and the Deep Blue Sea: The coastal zone in an Era of globalization. Estuarine, Coastal and Shelf Science, 96, 1-8, 2012.

Niehues, J. P. Sistema ambiental Ilha de Santa Catarina: ecossistemas dominantes, componentes e processos. 89 p. Florianópolis, Trabalho (Conclusão de Curso em Oceanografia) - Universidade Federal de Santa Catarina, 2014.

Nicolodi, J. L.; Asmus, M. L.; Polette, M.; Turra; A.; Seifert Júnior, C. A.; Mergen, B. O.; Stori, F. T.; Shinoda, D. C.; Mazzer, A. M. Avaliação dos zoneamentos ecológico-econômicos costeiros (ZEEC) no Brasil. In: Anais do X Encontro Nacional de Gerenciamento Costeiro - X ENCOGERCO, CIDEC Sul. FURG, 2017. p. 22-23.

Odum, H. T. Systems Ecology. New York: John Wiley, $1983.644 \mathrm{p}$. 
Odum, H. T.; Odum, E. C. A prosperous way down: Principles and policies. Colorado: University Press of Colorado, 2001.

Onetti, J. G.; Scherer, M. E. G.; Barragán, J. M. Integrated and ecosystemic approaches for bridging the gap between environmental management and port management. Journal of Environmental Management, 206, 615-624, 2018.

Regan, H. M.; Colyvan, M.; Burgman, M. A. A taxonomy and treatment of uncertainty for ecology and conservation biology. Ecological Applications, 12, 618-628, 2002.

Scherer, M. E. G.; Asmus, M. L. Ecosystem-Based Knowledge and Management as a tool for Integrated Coastal and Ocean Management: A Brazilian Initiative. In: Vila-Concejo, A.; Bruce, E.; Kennedy, D. M.; McCarroll, R. J. (Eds.). Proceedings of the 14th International Coastal Symposium (Sydney, Australia). Journal of Coastal Research, Special Issue, N. 75, Coconut Creek (Florida).

Scherer, M. E. G.; Asmus, M. L.; Onetti, J. G. Metodologia para identificação, com base ecossistêmica, dos aspectos e impactos ambientais significativos do Porto de Imbituba: Manual de aplicação. Relatório Técnico. Laboratório de Gestão Costeira Integrada (LAGECI), Universidade Federal de Santa Catarina, 2015. 78 p.

Silveira, V. M. M.; Silva, T. S.; Asmus, M. L.; Yamazaky, P. H. Unidades de planejamento com base ecossistêmica para ambientes costeiros. In: Anais do X Encontro Nacional de Gerenciamento Costeiro - X ENCOGERCO, CIDEC Sul. FURG, Rio Grande (RS), 2017. p. 148-149.

Simões, C. S.; Moura, D. V.; Rosa, C. da; Asmus, M. L.; Anello, L. A Percepção dos serviços ecossistêmicos e de suas vulnerabilidades frente a eventos atmosféricos extremos no Baixo Estuário da Lagoa dos Patos - Rio Grande/ RS-Brasil. In: Conde, D.; Polette, M.; Asmus, M. Risk, perception and vulnerability to Climate Change in wetland dependent coastal communities in the Southern Cone of Latin America. Final Report, IDRC Climate Change and Water Program, Project 6923001, 2015.
Tagliani, P. R. A; Asmus, M. L. O Programa de Manejo Integrado do Estuário da Lagoa dos Patos. In: Tagliani, P. R.; Asmus, M. (Ed.). Manejo integrado do Estuário da Lagoa dos Patos: uma experiência de gerenciamento costeiro no sul do Brasil. Rio Grande: Editora da FURG, 2011. 252 p., p. 27-39.

TEEB. The Economics of Ecosystems and Biodiversity. 2009. Disponível em: <http://www.teebweb.org/resources/ ecosystem-services/>.

Vasconcelos, V. V.; Martins-Júnior, P. P. Protótipo de sistema especialista em direito ambiental para auxílio à decisão em situações de desmatamento rural. NT-27. CETECMG, 2004. 80p.

Villasante, S.; Lopes, F. M.; Coll, M. The role of marine ecosystem services for human well-being: Disentangling synergies and trade-offs at multiple scales. Ecosystem Services, 17, 1-4, 2016.

Waterman, D. A. A Guide to Expert Systems. Addison-Wesley, 1986.

Yáñez-Arancibia, A. (Ed.). Cambio climático: Dimensión ecológica y socioeconómica. México: AGT Editor, S.A., 2013.

Yáñez-Arancibia, A.; Dávalos-Sotelo, R.; Day, J. W.; Reyes, E. Introduction to Ecological dimensions for sustainable socioeconomic development. In: Yáñez-Arancibia, A.; Dávalos-Sotelo, R.; Day, J. W.; Reyes, E. (Ed.). Ecological Dimensions for Sustainable Socio Economic Development. Southampton, UK: WIT Press, 2013. 636 p., p. 3-28. 